\title{
ZUR SEGMENTIERUNG UND TAXONOMIE DER GESCHICHTE DER FACHSPRACHEN UND IHRER UNTERSUCHUNG
}

\author{
1.
}

Bisher ist noch kein wesentlich systematischer Versuch unternommen worden, die Entstehungs- bzw. Entwicklungsgeschichte der Fachsprachen oder die Geschichte der bewussten Aussonderung dieser Fachsprachen oder die Geschichte der Beschäftigung mit den Fachsprachen im universellen Sinne systematisch darzustellen. Bisher wurden lediglich einzelne Ausschnitte dieser Geschichte/Geschichten untersucht, und zwar vorwiegend aus der Neuzeit. Fast unberührt bleibt die Frage der Fachsprachen auf dem „Gebiet“ der antiken Sprachen. Wir wissen darüber nicht viel im „Bereich“ der griechischen, der römischen oder der hebräischen Kultur, geschweige denn über die Fachsprachen in den Ländern des antiken Nahen Ostens sowie des alten China und alten Indien. Aber wir wissen auch nicht viel über die Fachsprachen und die Beschäftigung mit ihnen im Mittelalter. Etwas mehr wissen wir darüber aus späteren Zeiträumen, doch nur in Bezug auf einige wenige Länder.

Für die Geschichte der eigenen Fachsprachen interessierten sich wohl am ehesten die deutschen Forscher. Einige Elemente der detaillierten Entstehungsgeschichte der deutschen Fachsprachen bearbeiteten L. Drozd und W. Seibicke in dem Buch Deutsche Fach- und Wissenschaftssprache. Bestandsaufnahme - Theorie - Geschichte, das 1973 erschien. Die von ihnen dargestellte Geschichte vertieften und erweiterten J. Albrecht und R. Baum in der fast zehn Jahre später (1982) veröffentlichten Arbeit Fachsprache und Terminologie in Geschichte und Gegenwart. Aus der Feder deutscher Forscher stammt auch die bislang umfangreichste Darstellung der unterschiedlichen Richtungen und Konzepte in der Erforschung der Fachsprachen und Terminologien in Form der zweibändigen Sammelabhandlung Fachsprachen. Ein internationales Handbuch zur Fachsprachenforschung und Terminolo- 
giewissenschaft ${ }^{1}$, die 1998 unter wissenschaftlicher Redaktion von L. Hoffmann, H. Kalverkämper und E. Wiegand erschien. Dies ist nicht nur eine umfangreiche, sondern auch eine detaillierte Darstellung - die zwei Bände beinhalten insgesamt 272 Beiträge auf 2731 Seiten. In derselben Reihe HSK Bd. 23 Histoire linguistique de la Romania/Romanische Sprachgeschichte ${ }^{2}$ (Ernst et al. 2006) wurden auch einige Aspekte der Geschichte einiger romanischer Fachsprachen dargestellt. Einige Beträge zur Geschichte der jeweiligen Fachsprachen sind auch in der Zeitschrift Fachsprachen - International Journal of Specialized Communication ${ }^{3}$ zu finden.

Das Verdienst, als erste polnische Monographie anerkannt zu werden, die den Fachsprachen gewidmet ist und vom linguistischen Standpunkt aus geschrieben wurde, gebührt dem Werk von S. Gajda Wprowadzenie do teorii terminu [Einführung in die Theorie des Fachterminus], das 1990 veröffentlicht wurde. Es ist jedoch gleich hinzuzufügen, dass diese Monographie nur insoweit als eine den Fachsprachen gewidmete Arbeit anzuerkennen ist, als die Fachtermini für gewisse (lexikalische) Bestandteile der Fachsprachen anzuerkennen sind, anders gesagt: Als eine sich auf Fachsprachen beziehende Monographie kann (und soll) sie vom Standpunkt des zeitgenössischen, bereits angesprochenen, allgemeinen Verständnisses der Fachsprachen gelten. Die Arbeit von S. Gajda ist auch die erste polnische Monographie, die einen linguistischen Abriss der Geschichte der nur Versuche, Entstehungs- bzw. Entwicklungsgeschichte des Fachwortschatzes auf manchen Gebieten der Praxis und/oder der Wissenschaft darzustellen.

Interessant ist in diesem Zusammenhang, dass bis dato weder in der deutschsprachigen noch in der polnischen Linguistik der Versuch unternommen wurde, Kriterien (Vorgehensweisen) für eine systematische und detaillierte Bearbeitung der Entstehungs- und Entwicklungsgeschichte von Fachsprachen - auf der einen Seite, sowie der Geschichte der Untersuchung von Fachsprachen - auf der anderen Seite, ausgearbeitet wurden.

Im Folgenden sollen kurz einige Aspekte, die, meines Erachtens, berücksichtigt werden müssen, bevor überhaupt mit der Rekonstruktion der Entstehungs- bzw. Entwicklungsgeschichte von Fachsprachen sowie der Beschäftigung mit ihnen begonnen werden kann. Beginnt man zudem mit der Darstellung der Geschichte der Fachsprachen, sollte man sich zunächst deren ontologische Aspekte bewusst machen, d. h. klären, was sie sind und was sie nicht sind. Des Weiteren soll man sich bewusst werden, was Fachsprachen beinhalten und was sie nicht beinhalten. Nicht zuletzt muss auch die Frage beantwortet werden, wozu Fachsprachen dienen können, welche Funktionen mit ihnen erfüllt werden können.

\footnotetext{
${ }^{1}<$ http://www.reference-global.com/doi/book/10.1515/9783110111019.1> (Zugriff am 17.08.2011)

${ }^{2}<$ http://www.reference-global.com/doi/book/10.1515/9783110146943.1> (Zugriff am 17.08.2011)

${ }^{3}<$ http://www.fachsprache.net/> (Zugriff am 17.08.2011)
} 
2.

Gehen wir also zuerst dieser Fragen nach. ${ }^{4}$ Fangen wir dabei an, dass am Anfang linguistischer Überlegungen der konkrete Mensch und seine konkrete Sprache steht, stehen soll. Und dies deswegen, da, im ontologischen Sinne, nur die Sprache eines konkreten Menschen konkret ist, da sie eine reale (konkrete) Ausstattung des menschlichen Gehirns ist. So wird hier der Ausdruck „Sprache“ zuerst auf „die konkrete Sprache eines konkreten Menschen" bezogen, und in dieser Bedeutung als „Idiolekt" bezeichnet. Erst auf der Grundlage von Beobachtungen und Vergleichen konkreter Menschen und ihrer Idiolekte können Modelle/Konstrukte gebildet werden. In diesem Sinne kann sich der Ausdruck „Sprache“ auch auf ein linguistisches (mentales) Konstrukt beziehen, das entweder als Schnittmenge oder Vereinigung von Merkmalen bestimmter Idiolekte anzusehen ist. Diese Konstrukte werden in der anthropozentrischen Sprachentheorie als Polylekte bezeichnet. Dies hat bereits sehr deutlich F. Grucza an verschieden Stellen zum Ausdruck gebracht:

In Wirklichkeit existiert keine menschliche Sprache selbstständig. Wirklich existieren menschliche Sprachen immer nur in Form gewisser Bestandteile bestimmter (miteinander vernetzter) Eigenschaften konkreter Menschen. Ganz allgemein kann man diese Eigenschaften die „sprachlichen Eigenschaften" nennen. Es handelt sich um diejenigen Eigenschaften, die es Menschen ermöglichen, einerseits bestimmte Äußerungen zu formulieren, sie mit Bedeutungen zu ,versetzen" und die Formen der Äußerungen mündlich bzw. schriftlich zu realisieren, und andererseits Äußerungen anderer Menschen zu empfangen, zu identifizieren, zu interpretieren und zu verstehen. (...) Im engeren Sinne sind wirkliche menschliche Sprachen bestimmte Bereiche des praktischen Wissens konkreter Menschen. (...) Doch gleichgültig, ob wir sie im engeren oder weiteren Sinne betrachten, wirkliche menschliche Sprachen sind keine selbständigen Objekte. Als selbständige Objekte funktionieren nur die Inhaber wirklicher Sprachen - die konkreten Menschen (Individuen). Zwar können auch externalisierte sprachliche Äußerungen (Texte) als (relativ) selbständige Objekte angesehen werden, doch besitzen sie im Grunde genommen keine funktionale sprachliche Selbständigkeit (F. Grucza 2002: 233f.).

Diese Beobachtungen beziehen sich in gleichem Maße auf die Gemeinsprachen (Gemeinlekte) wie auch auf die Fachsprachen (Fachlekte). Daraus ergibt sich, dass der Ausdruck „Fachsprache“ zuerst als die konkrete Fachsprache eines konkreten Menschen, also als sein Fachidiolekt verstanden wird. Eine (konkrete) Fachsprache, wie jede andere (konkrete) Sprache eines (konkreten) Menschen, ist seine immanente Eigenschaft, genau gesagt, eine Eigenschaft seines Gehirns. In dem Sinne ist es eine Fachsprache eines konkreten Menschen, eines konkreten Fachmanns, ein konkreter Wirklichkeitsbereich. Dies bedeutet zuerst, dass Fachsprachen weder abstrakte Systeme noch autonome Gebilde sind (sie sind im Übrigen auch keine konkreten Systeme; genauer dazu s. S. Grucza 2008).

\footnotetext{
${ }^{4}$ Ausführlicher dazu s. F. Grucza 1988; 1993; 2010; S. Grucza 2010.
} 
Der Ausdruck „Fachidiolekt” kann sich aber auch auf ein Konstrukt beziehen, das entweder als Schnittmenge oder Vereinigung von Fachidiolekten der in Betracht gezogenen Fachleute konstruiert werden kann. Dieses Konstrukt ist als „Fachpolylekt" zu bezeichnen (s. Schema 1). Hinzugefügt sei noch, dass Fachpolylekte, aufgefasst als Vereinigung von Fachidiolekten der in Betracht gezogenen Fachleute, in der Rekonstruktion und Beschreibung (rekonstruktiver Erforschung) grammatischer Komponenten von Fachsprachen (Fachidiolekten), und Fachpolylekte aufgefasst als Vereinigung von Fachidiolekten der in Betracht gezogenen Fachleute in der Rekonstruktion und Beschreibung lexikalischer Komponenten von Fachsprachen (Fachidiolekten) in Betracht gezogen werden (s. Schema 2). Sowohl Fachidiolekte als auch Fachpolylekte müssen von deren Beschreibungen (Modellen) unterschieden werden. Darauf hat schon sehr deutlich F. Grucza (1994: 21) hingewiesen (s. auch S. Grucza 2008).

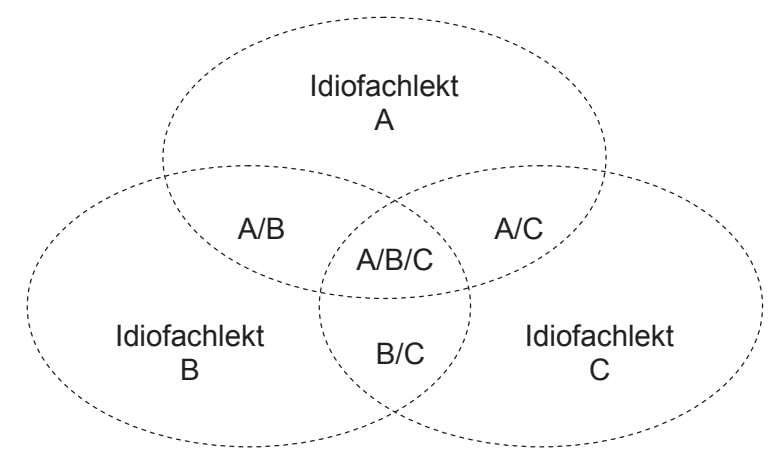

Schema 1. Polylektale Teile (Schnittmengen) der Menge dreier Idiofachlekte

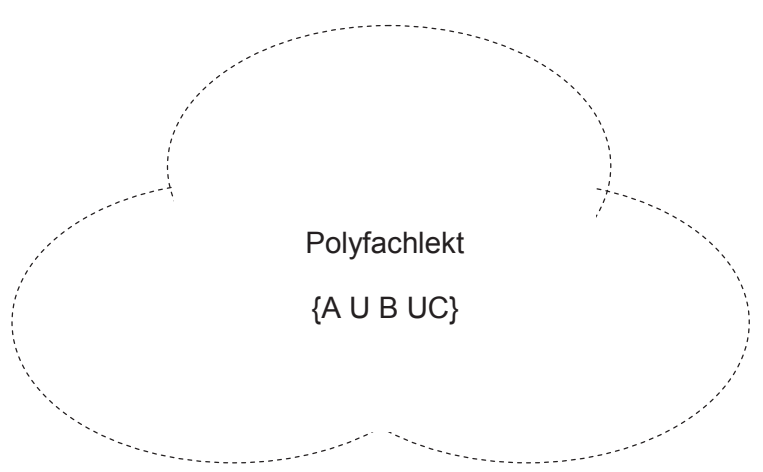

Schema 2. Polylektale Teile (Vereinigung) der Menge dreier Idiofachlekte 
Aus dem oben Gesagten ergibt sich, dass unter dem komponentalen Gesichtspunkt Fachsprachen/Fachidiolekte gegenüber der jeweiligen Gemeinsprache (dem Gemeinidiolekt) keine autonomen (vollständigen) Sprachen sind. Unter diesem Gesichtspunkt ist jede Fachsprache (jeder Fachidiolekt) mehr oder weniger mit der Gemeinsprache (dem Gemeinidiolekt) verbunden und zwar so, dass sich die Menge der Fachsprachenkomponenten und die Menge der Gemeinsprachenkomponenten überschneiden, d.h. eine Schnittmenge bilden. Vor allem aber FachsprachenPhonemik und Fachsprachen-Grammatik decken sich in der Regel fast vollständig mit der Phonemik und Grammatik der entsprechenden Gemeinsprache. Dafür überschneiden sich die Lexik-Komponenten der Fachsprachen und der Gemeinsprache nur zum Teil - je nach Art der Fachsprache in unterschiedlichem Grade. Beide, die Fachsprachen und die Gemeinsprache, haben auch ,eigene“ Textmuster-Komponenten. Die Terminologie-Komponenten sind demgegenüber nur für Fachsprachen charakteristisch.

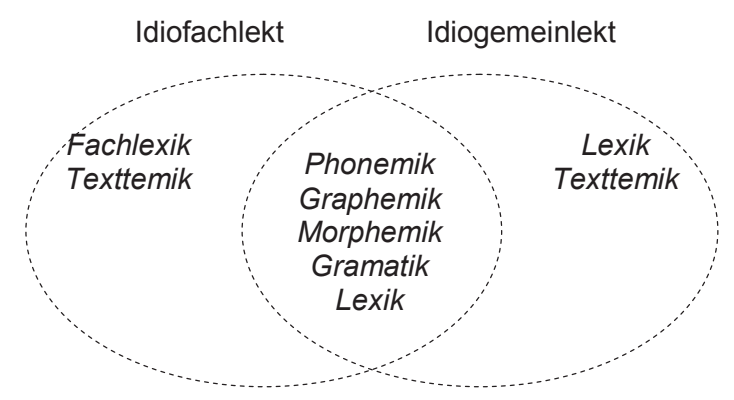

Schema 3. Der gemeinsame Bereich der konstitutiven Elemente des Idiofach- und Idiogemeinlekts

Es ist zu bemängeln, dass eine holistische Herangehensweise an die Fachsprachen immer noch sehr zurückhaltend stattfindet, obwohl schon Ende der 60er Jahre D. Möhn in einem Beitrag unter dem übrigens für diese Zeiten interessanten Titel Fach- und Gemeinsprache. Zur Emanzipation und Isolation der Sprache eindeutig betont, dass die wissenschaftlichen Arbeiten als bahnbrechend angesehen werden können, die die Terminologien lediglich als Bestandteile von ganzen Fachsprachen betrachten:

Ein bemerkenswerter Fortschritt wird in Arbeiten deutlich, die Fachwörter nicht isoliert, sondern als Teil eines Ganzen sehen, d. h. die Fachsprachen nicht auf die Terminologie beschränken, sondern ihnen gesamtsprachliche Eigenschaften zuerkennen (...) (Möhn 1968: 316).

Ähnlich wie D. Möhn hat generell Fachsprachen auch L. Hoffmann (1976) betrachtet. Für ihn bedeutet die Analyse der spezifischen Merkmale der Fachsprachen 
auf den einzelnen sprachlichen Ebenen und Zeichenebenen, d. h. die Analyse der Grapheme, Phoneme, Morpheme, grammatischen Kategorien, Lexeme, Syntagmen, Phrasen und Sätze:

Fachsprache - das ist die Gesamtheit aller sprachlichen Mittel, die in einem fachlich begrenzbaren Kommunikationsbereich verwendet werden, um die Verständigung zwischen den in diesem Bereich tätigen Menschen zu gewährleisten (Hoffmann 1976: 170).

Abgesehen von den offensichtlichen Mängeln der Fachsprachen-Konzeption von L. Hoffmann (vor allem die Betrachtung von (Fach)Sprachen als einer Menge sprachlicher Mittel), muss doch darauf hingewiesen werden, dass die zitierte Äußerung von L. Hoffmann davon zeugt, dass einige deutsche Sprachwissenschaftler bereits Mitte der 70er Jahre des 20. Jh. eindeutig klar ihre Überzeugung darüber zum Ausdruck brachten, dass der Bereich der Realität, die als „Fachsprache“ bezeichnet wurde, nicht nur Fachtermini (Fachlexik) umfasse, sondern darüber hinaus alle anderen Bestandteile, die den Sprachen im Allgemeinen eigen sind - L. Hoffmann gebrauchte dafür die Formulierung Gesamtheit der sprachlichen Mittel.

Gleichzeitig sind die meisten Vertreter der Fachsprachenlinguistik in den 70er und 80er Jahren des 20Jh. in ihren Fachsprachen-Bestimmungen unter einem sehr starken Einfluss der Systemlinguistik, was in der Betrachtung von Fachsprachen als besondere Sprachsysteme, die durch Sprachregeln und Sprachzeichen konstituiert werden, zum Ausdruck kommt. Dies ist nicht nur an der oben zitierten Aussage von L. Hoffmann sondern auch an vielen anderen abzulesen:

Die Fachsprachen sind Bestandteile natürlicher Sprachsysteme, in denen eine endliche Menge von sprachlichen Zeichen und Regeln für deren Kombination verfügbar ist. Das bedeutet, dass in den Fachsprachen die gleichen Sprachelemente verwendet werden, wie in der Gemeinsprache, Umgangssprache oder Literatursprache. Allerdings werden - bedingt durch die andersgearteten Kommunikationsstrukturen (...) - morphologische, syntaktische und lexikalische Sprachelemente in von der Gemeinsprache abweichender Weise eingesetzt. Damit wird bereits angedeutet, dass wir nicht geneigt sind, Fachsprache mit Fachwortschatz oder gar Terminologie gleichzusetzen, das heißt, wir erkennen den Fachsprachen in gewissen Grade autonome strukturelle Eigenschaften zu (Bausch, Schewe, Spiegel 1976: 12).

3.

Im Rahmen jeder hoch entwickelten Gesellschaft erfolgte zuerst die Herausbildung von Fachsprachen, die der Ausübung von bestimmten Bereichen der praktischen Arbeit dienten, d.h. mit bestimmten Handwerks-, Medizin-, und/oder Baukunstarten verbunden waren. Diese Fachsprachen nenne ich hier praktische Fachsprachen. Wenig später folgte innerhalb dieser Gesellschaften auch immer eine Entwicklung von Fachsprachen, die der Erkenntnis oder der Gesellschaftsorganisation dienten. Diese Fachsprachen bezeichne ich hier als kognitive Fachsprachen. 
Die Rolle der Fachsprachen erschöpft sich nicht nur in der kommunikativen Funktion innerhalb verschiedener Fachgemeinschaften. Fachsprachen sind aber nicht ausschließlich kommunikative Werkzeuge „ihrer“ Fachgemeinschaften. Sie sind auch, und vielleicht auch in erster Linie, kognitive Werkzeuge der jeweiligen Fachgemeinschaften, da sie ihren Mitgliedern als Werkzeuge der Facherkenntnis dienen. Fachsprachen erfüllen eine kommunikative Funktion, da dank ihnen Fachtexte produziert und rezipiert (verstanden) werden können, wodurch auf der Seite des Textproduzenten Fachwissen ausgedrückt und auf der Seite des Textrezipienten dieses aufgebaut (konstituiert) werden kann. Fachsprachen erfüllen eine kognitive Funktion, da dank ihnen neues Fachwissen gebildet und altes umorganisiert werden kann.

Nach M. Tarantino (2004) hat schon Aristoteles auf die epistemische Rolle von Sprachen hingewiesen:

Aristotle names the discourse of scientific inquiry Episthemonikos, i.e., knowledge making discourse. He associates this speech type with the purpose of understanding, explaining, classifying natural processes as well as establishing inter-relations between phenomena, objects and organisms observable in the physical world

Dass zwischen der Erkenntnis und menschlichen Sprachen eine tief greifende, gegenseitige Abhängigkeit besteht, wurde auch A. von Humboldt hervorgehoben:

Durch die gegenseitige Abhängigkeit des Gedankens, und des Wortes voneinander leuchtet es klar ein, dass die Sprachen nicht eigentlich Mittel sind, die schon erkannte Wahrheit darzustellen, sondern weit mehr, die vorher unerkannte darzustellen. Ihre Verschiedenheit ist nicht eine von Schällen und Zeichen, sondern eine Verschiedenheit der Weltansichten selbst (Humboldt 1968: 27).

Einen Hinweis darauf, dass besonders Fachsprachen der Erkenntnis dienen, lässt sich auch aus den Aussagen von D. Möhn und R. Pelka (1984: 26) rekonstruieren:

Wir verstehen unter Fachsprache heute die Variante der Gemeinsprache, die der Erkenntnis und begrifflichen Bestimmung fachspezifischer Gegenstände sowie der Verständigung über der sie dient und damit den kommunikativen Bedürfnissen im Fach allgemein Rechung trägt. ${ }^{5}$

Dass Fachsprachen als eines der wichtigsten Mittel der zivilisatorischen Entwicklung ihrer (Fach)Gemeinschaften betrachtet werden können oder sogar müssen, dass der Entwicklungsstand der jeweiligen Fachsprache sowohl als Zeugnis, als auch

\footnotetext{
${ }^{5}$ Sehen wir hier davon ab, dass der offensichtliche Mangel solcher Betrachtung der Fachsprachen darin liegt, dass wenn Fachsprachen Varianten im gegenseitigen Verhältnis und im Bezug auf die Gemeinsprache wären, dann könnte man genauso über dieselben Realitätsbereiche sprechen, auf die sich sowohl Gemeinsprache als auch die Fachsprachen beziehen. Bekanntlich ist es nicht möglich, das Wissen aus dem Gebiet Chemie genauso exakt (adäquat) darstellen, wenn man sich dabei der Gemeinsprache oder der Fachsprache der Linguistik bedient, wie dies möglich ist, wenn man sich der Fachsprache der Chemie bedient. Da dies nicht möglich ist, sind die einzelnen Fachsprachen keine Varianten weder anderer Fachsprachen, noch keiner Gemeinsprache. Dies betrifft selbstverständlich gleichermaßen die praxisorientierten wie die kognitiven Fachsprachen, sowie beide Typen im engen, weiteren und weitesten Sinne.
} 
als Maßstab der zivilisatorischen Entwicklung der jeweiligen (Fach)Gemeinschaft aufgefasst werden kann, hat wohl zum ersten Mal expressis verbis F. Grucza (2002; 2004) zum Ausdruck gebracht. Der Ausgangspunkt für seine Überlegungen ist die Feststellung, dass sowohl das Schaffen der Fachkenntnisse als auch die Formulierung der Fachtexte, in denen die Fachleute ihre Kenntnisse zum Ausdruck bringen, allein mit Hilfe der Fachsprachen möglich ist. Auf der funktionalen Ebene erfüllen die Fachsprachen die Funktion der „Werkzeuge“ für die Beschreibung und Schöpfung der Wirklichkeit, d. h. der Bildung des Fachwissens. Da die Fachsprachen auf der Zeichenebene gewissermaßen bestimmte Koeffizienten „nachbilden“, die die Wirklichkeit erschaffen, erfüllen sie auch kognitive Funktionen:

\begin{abstract}
Wie andere Arten von Fachsprachen, so auch die uns hier interessierenden Fachsprachen erfüllen nicht nur kommunikative Funktionen, sondern auch wichtige kognitive Funktionen. In jedem Fall sind ziemlich große Teile verschiedenartigen Fachwissens eine Art Derivate der „Kenntnis“ entsprechender Fachsprachen und/oder fachlicher Äußerungen über die betreffenden Bereiche der Wirklichkeit. (...) Viele Elemente des Wissens der einzelnen Menschen wie auch der Gemeinschaften über die gegebene Wirklichkeit oder Elemente der Verständnis des Wissens ,stammen“ aus der Kenntnis der diese Wirklichkeit betreffenden (Fach-)Sprachen, und nicht unbedingt aus der Erfahrung und/oder den Gedankengängen. Die Qualität dieser Elemente des sowohl allgemeinen als auch Fachwissens einzelner Menschen und Gemeinschaften hängt von der Qualität der betreffenden (Fach-)Sprachen ab. Hingegen hängt die Qualität der letzteren - im überwiegenden Maße - von dem Grad des „Erkennens“ der durch die Fachsprachen denotierten Wirklichkeit. (F. Grucza 2004: 22)
\end{abstract}

M.A. Krąpiec (1995: 32) fügt hinzu, dass diese Verbindung eine Art Rückkoppelung ist, da auf der einen Seite die Erkenntnis der Wirklichkeit in einer bestimmten Weise durch Sprache determiniert wird, und auf der anderen Seite die Wirklichkeit sich in gewissem Masse in der Sprache „,widerspiegelt“. In der Erläuterung dieser Relation beruft sich M.A. Krąpiec auf das Prinzip der übergeordneten Wirkung, das durch Thomas von Aquin so formuliert wurde: „Instrumentum non agit, nisi per aliquid sibi proprium dispositive operatur et effectum principalis agentis". Zusammenfassend äußerte sich dazu M.A. Krąpiec (1995: 35) folgendermaßen:

Sprache, die auf bedeutende und unzertrennliche Weise mit dem Gedanken verflochten ist, bildet zusammen mit ihm eine eigenartige Ganzheit bei dem Menschen, die eine Verbindung von SubjektObjekt-Relationen ist. ${ }^{6}$

Fassen wir kurz zusammen: (1) Fachsprachen dienen der Facherkenntnis, Facherkenntnis eo ipso ist nur über Fachsprachen möglich; (2) Fachsprachen dienen der Fachkommunikation, Fachkommunikation eo ipso ist nur über Fachsprachen möglich; (3) Fachsprachen sollen unter funktionalen Gesichtspunkten gegenüber der jeweiligen Gemeinsprache als autonom aufgefasst werden; (4) Fachsprachen sind unter ontologischen Gesichtspunkten gegenüber der jeweiligen Gemeinsprache keine autonomen Sprachen (ausführlicher dazu s. S. Grucza 2009).

\footnotetext{
${ }^{6}$ Mühlhölzer 1993; Cohen1993; Simonnæs 2009.
} 
4.

Beginnt man mit der Darstellung der Geschichte der Fachsprachen, ist es ebenso bedeutend, sich dessen bewusst zu sein, dass die Entstehungs- bzw. Entwicklungsgeschichte der Fachsprachen nicht in dem Moment begann, in dem ihre Existenz zum ersten Mal mit Texten belegt wurde, auch nicht dann, als sie zum ersten Mal als Fachsprachen bezeichnet wurden. Darüber hinaus sollte man sich auch darüber klar sein, dass die Geschichte der Beschäftigung mit den Fachsprachen nicht in dem Moment begann, in dem zum ersten Mal Ergebnisse diesbezüglicher Überlegungen und/oder Forschungen veröffentlicht wurden, anders gesagt, diese Geschichte begann nicht erst dann, als die Forscher, die sich zum ersten Mal mit den Fachsprachen befassten, deren Existenz mit Texten belegt haben. Die Entstehungs- bzw. Entwicklungsgeschichte von Fachsprachen und daher auch die Geschichte der Beschäftigung mit den Fachsprachen müssen viel weiter „nach hinten“ verschoben werden. Darüber hinaus sollte meines Erachtens sowohl die eine als auch die andere Geschichte in verschiedenen Etappen und Teile (partielle historische Abschnitte) eingeteilt werden, und zwar zunächst je nach dem, ob der Untersuchungsgegenstand praxisorientierte oder kognitive Fachsprachen sind, je nach dem muss noch eine ganze Reihe von Aspekten berücksichtigt werden. Zusammenfassend ergibt sich aus dem bis jetzt angesammelten Wissen über die Fachsprachen, dass, wenn in der Zukunft mit geschichtsorientierten Beschreibungen der Fachsprachen begonnen wird, dann eine weitgehende Taxonomie vorgenommen werden sollte, und zwar sowohl in Bezug auf die Entstehungsgeschichte der Fachsprachen als auch auf die Geschichte der Beschäftigung mit ihnen. Unter anderem sollten folgende Aspekte voneinander unterschieden und / oder getrennt werden:

1. die Entstehungs- bzw. Entwicklungsgeschichte der Fachsprachen von der Geschichte der Beschäftigung mit ihnen;

2. die Geschichte der nichtbewussten Beschäftigung mit den Fachsprachen von der Geschichte der bewussten Beschäftigung mit ihnen;

3. die Geschichte der vorwissenschaftlichen Beschäftigung mit den Fachsprachen von der Geschichte der wissenschaftlichen Beschäftigung mit ihnen;

4. die Entstehungs- bzw. Entwicklungsgeschichte der Fachsprachen im engen Sinne des Wortes, der sich auf den Wortschatz (Terminologie) bezieht, von der Entstehungsgeschichte der Fachsprachen im weiteren Sinne des Wortes, der sich auch auf die Grammatik bezieht, und von der Entstehungsgeschichte der Fachsprachen im noch weiteren Sinne des Wortes, der sich auch auf die Texte bezieht;

5. die Geschichte der Beschäftigung mit den Fachsprachen im engen Sinne des Wortes von der Geschichte der Beschäftigung mit den Fachsprachen im weiteren Sinne und von der Geschichte der Beschäftigung mit den Fachsprachen im weitesten Sinne; 
6. die Entstehungsgeschichte der praxisorientierten Fachsprachen (im engen, weiteren und weitesten Sinne) von der Entwicklungsgeschichte der kognitiven Fachsprachen (im engen, weiteren und weitesten Sinne);

7. die Geschichte der praxisorientierten Beschäftigung mit den Fachsprachen, z. B. mit dem Ziel, diese zu ordnen und zu präzisieren, von der Geschichte der wissenschaftlichen Beschäftigung mit ihnen, $d$. $h$. der Beschäftigung schlechthin mit dem Ziel, die Fachsprachen zu erkunden, kurz gesagt, die Geschichte des angewandten Interesses an ihnen von der Geschichte der Beschäftigung mit ihnen vom Standpunkt der reinen Wissenschaft;

8. die Geschichte der Beschäftigung mit den Fachsprachen hinsichtlich ihrer Kommunikations-/Ausdrucksfunktionen oder -rollen von der Geschichte der Beschäftigung mit ihnen hinsichtlich ihrer kognitiven (Erkenntnis) funktionen oder -rollen;

9. die Geschichte der Beschäftigung mit Fachsprachen seitens der Vertreter anderer Disziplinen von der Geschichte der Beschäftigung mit ihnen seitens der Linguisten;

10. die Geschichte der Beschäftigung mit den Fachsprachen als gewissen Bestandteilen der Errungenschaften der einen oder anderen Sprachgemeinschaft, also mit den „nationalen“ Fachsprachen, von der Geschichte der Beschäftigung mit ihnen in universeller Dimension;

11. die Geschichte der institutionalisierten Beschäftigung mit den Fachsprachen aus dem Bedürfnis heraus, diese zu vereinheitlichen und zu normieren von der Geschichte der institutionalisierten (akademischen) Beschäftigung mit ihnen vom Standpunkt der Erkenntnis.

Aus dem oben Gesagten geht hervor, dass vor der am Anfang dargestellten Taxonomie der Geschichte der Beschäftigung mit den Fachsprachen noch die Geschichte der Beschäftigung mit den Fachsprachen im weiteren textuellen Kontext von der Geschichte der Beschäftigung mit den Fachsprachen im weiteren diskursiven Kontext unterschieden werden sollte. Mit dem letzteren werde ich mich hier nicht näher befassen, denn in den Vordergrund der diskursiven Textlinguistik werden vor allem Fragen nach der Interaktion gestellt, die für das Thema der vorliegenden Arbeit keine wesentliche Rolle spielen.

\section{5.}

Der hier präsentierte Ansatz zur Segmentierung und Taxonomie der Geschichte der Fachsprachen und ihrer Untersuchung geht einen anderen Weg als die meisten bisherigen Vorschläge. Er unterscheidet er sich auch in der Art und Weise der Periodisierung der Fachsprachengeschichte. So schlägt Th. Roelcke (1999: 160) die Unterteilung der deutschen Fachsprachengeschichte in drei Perioden vor: die erste Periode, mittelalterliche Fachsprachen, vom 8. Jh. bis etwa zur Mitte des 14. Jh., die zweite Periode, frühneuzeitliche Fachsprachen, vom 14. Jahrhundert bis zum Ende 
des 17. Jh., die dritte Periode, neuzeitliche Fachsprachen, vom 18. bis $20 \mathrm{Jh}$. Für diese temporale Gliederung führt er folgende Argumente an:

Die Vorschläge, die zur Periodisierung der Geschichte der deutschen Sprache seit dem 17. Jahrhundert vorgelegt wurden, berücksichtigen zwar unter dem Kriterium sprachlicher Varietäten wiederholt auch die Entwicklung von Fachsprachen, werden dabei jedoch deren Besonderheiten kaum gerecht, da sie diese der Gesamtentwicklung des Deutschen und seiner Varietäten unterordnen. Die bis in die Gegenwart hinein gebräuchlichen Einteilungen nach Jacob Grimm (Althochdeutsch 6001100, Mittelhochdeutsch 1100-1500, Neuhochdeutsch seit 1500) und Wilhelm Scherer (althochdeutsche Zeit 750-1050, mittelhochdeutsche Zeit 1050-1350, frühneuhochdeutsche Zeit 1350-1650, neuhochdeutsche Zeit seit 1650), erweisen sich für eine Gliederung der deutschen Fachsprachengeschichte nur als bedingt tauglich, zumal sie anhand anderer Kriterien gewonnen wurden. Im folgenden wird die deutsche Fachsprachengeschichte in drei Perioden gegliedert, die zum einen die Kulturgeschichte des europäischen Sprachraums im allgemeinen und zum anderen den Bestand an verschiedenen Fachsprachen im deutschen Sprachraum widerspiegeln.

Aus meiner Sicht kann die Geschichte der wissenschaftlichen Untersuchungen zu Fachsprachen in unterschiedliche Etappen und historische Teilabschnitte eingeteilt werden - mindestens aber in vier Zeiträume. Den ersten bezeichne ich als Zeitraum von der nicht-linguistischen zur linguistischen Beschäftigung mit den Termini, d. h. mit den Fachsprachen im engsten Sinne; den zweiten als Zeitraum von der terminologischen zur syntaktischen Fachsprachenlinguistik; den dritten als Zeitraum von der syntaktischen zur textuellen Fachsprachenlinguistik; und den vierten als Zeitraum von der textuellen zur kommunikativen Fachsprachenlinguistik (eine umfangreichere Charakteristik dieser Zeiträume habe ich in der Arbeit S. Grucza 2008 präsentiert).

Vom Standpunkt des weitesten Verständnisses der Fachsprachen erscheint die Geschichte der Beschäftigung mit ihnen:

1. als ein Prozess der Einbindung von immer neuen Bestandteilen der die authentischen Fachsprachen bildenden Realität, die als „Fachsprache“ bezeichnet wird, in den Interessensbereich der Wissenschaft;

2. als ein Prozess der Entdeckung und gleichzeitig Berücksichtigung von immer neuen Aspekten oder Funktionen der Fachsprachen in deren Forschung;

3. als ein Prozess der immer weiteren Entfernung der Fachsprachenlinguistik von der „klassischen“ Terminologie und der immer größeren Verselbständigung der ersteren, und schließlich;

4. ein Prozess der immer tiefergehenden linguistischen Spezialisierung der Terminologie.

\section{Literatur}

Albrecht J., Baum R. (1982): Fachsprache und Terminologie in Geschichte und Gegenwart. Tübingen: Narr. Bausch, K.-H., Schewe, W.H., Spiegel H.-R. (1976): Fachsprachen. Terminologie, Struktur, Normung. Normenkunde. Heft 4. Berlin. 
Cohen, L.J. (1993): Philosophy of language and ontology. In: M. Dascal, D. Gerhardus, K. Lorenz, G. Meggle (Hrsg.): Sprachphilosophie. Ein internationales Handbuch zeitgenössischer Forschung. Berlin-New York: de Gruyter. Bd. 1, S. 1729-1738.

Drozd L., Seibicke, W. (1973): Deutsche Fach- und Wissenschaftssprache. Bestandsaufnahme-Theorie - Geschichte. Wiesbaden: Oscar Brandstetter Verlag.

Ernst G., Gleßgen, M.-D., Schmitt, Ch., Schweickard, W. (Hrsg.) (2006): Manuel international d'histoire linguistique de la Romania/Ein internationales Handbuch zur Geschichte der romanischen Sprachen. Berlin-New York: de Gruyter.

Gajda, S. (1990): Wprowadzenie do teorii terminu. Opole: Wyższa Szkoła Pedagogiczna im. Powstańców Śląskich w Opolu.

Grucza, F. (1988): Zum Begriff der Sprachkompetenz, Kommunikationskompetenz und Kulturkompetenz. In: CHLOE. Beihilfe zum Daphnis 7. Daß eine Nation die Andere verstehen möge. Festschrift für Marian Szyrocki zu seinem 60. Geburtstag. Amsterdam, S. 309-331.

Grucza, F. (1993): On concepts of human language(s), the subject and goals of linguistics. In: J. Darski, Z. Vetulani (Hrsg.): Sprache - Kommunikation - Informatik, Bd. 1. Tübingen: Niemeyer, S. 3-13.

Grucza, F. (1994): O językach specjalistycznych (= technolektach) jako pewnych składnikach rzeczywistych języków ludzkich. In: F. Grucza, Z. Kozłowska (Hrsg.): Języki specjalistyczne. Warszawa: Euro-Edukacja. S. 7-27.

Grucza, F. (2002a): Zu den Forschungsgegenständen der Linguistik und der Glottodidaktik - Zum Wesen menschlicher Sprachen und zu ihren Funktionen. In: H. Barkowski, R. Faistauer (Hrsg.): In Sachen Deutsch als Fremdsprache: Sprachenpolitik und Mehrsprachigkeit, Unterricht, interkulturelle Begegnung. Festschrift für Hans-Jürgen Krumm. Hohengehren: Schneider Verlag, S. 231-244.

Grucza, F. (2002b): Języki specjalistyczne - indykatory i/lub determinatory rozwoju cywilizacyjnego. In: J. Lewandowski (Hrsg.): Języki specjalistyczne 2. Problemy technolingwistyki. Warszawa: KJS UW, S. 9-26.

Grucza, F. (2004): O językach dotyczących europejskiej integracji i Unii Europejskiej i potrzebie ukonstytuowania ogólnej lingwistyki języków specjalistycznych. In: J. Lewandowski (Hrsg.): Języki specjalistyczne 4. Leksykografia terminologiczna - teoria i praktyka. Warszawa: KJS UW, S. 9-51.

Grucza, F. (2010): Zum ontologischen Status menschlicher Sprachen, ihren Funktionen, den Aufgaben der Sprachwissenschaft und des Sprachunterrichts. In: Kwartalnik Neofilologiczny 3/2010, S. 257-274.

Grucza, S. (2007): Od lingwistyki tekstu do lingwistyki tekstu specjalistycznego. Warszawa: EuroEdukacja.

Grucza, S. (2008): Lingwistyka języków specjalistycznych. Warszawa: Euro-Edukacja.

Grucza, S. (2009): Zur kognitiven Funktion von Fachsprachen und zur Geschichte ihrer Erforschung. In: E. Białek, J. Rzeszotnik, E. Tomiczek (Hrsg.): Auf der Suche nach Humanista. Dresden-Wrocław: Atut/ Neisse Verlag, S. 153-165.

Grucza, S. (2010): Główne tezy antropocentrycznej teorii języków. In: Lingwistyka Stosowana. Applied Linguistics. Angewandte Linguistik 2/2010, S. 41-68.

Hoffman, L. (1976): Kommunikationsmittel Fachsprache: eine Einführung. Berlin: Akademie Verlag.

Hoffmann L., Kalverkämper, H., Wiegand, H.E. (Hrsg.) (1998): Fachsprachen. Ein internationales Handbuch zur Fachsprachenforschung und Terminologiewissenschaft. Bd. 1 i 2. Berlin-New York: de Gruyter.

Humboldt, W. von (1968): Über das Vergleichende Sprachstudium. In: A. Leitzmann (Hrsg.): W. Humboldt von 1903-1936. Gesamelte Schriften. Bd. IV., Berlin.

Juszkiewicz, U. (1982): Z historii i teorii prac nad polską terminologią techniczną. In: Poradnik Językowy 1982, H. 2, S. 82-88.

Krąpiec, M.A. (1995): Dzieła XIII. Język i świat realny. Lublin: RW KUL. 
Möhn, D. (1968): Fach- und Gemeinsprache. Zur Emanzipation und Isolation der Sprache. In: W. Mitzka (Hrsg.): Wortgeographie und Gesellschaft. Berlin: de Gruyter, S. 23-42.

Möhn, D., Pelka, R. (1984): Fachsprachen. Eine Einführung. Tübingen: Niemeyer.

Mühlhölzer, F. (1993): Sprachphilosophie in der Wissenschaftstheorie. In: M. Dascal, D. Gerhardus,

K. Lorenz, G. Meggle (Hrsg.): Sprachphilosophie. Ein internationales Handbuch zeitgenössischer Forschung. Berlin-New York: de Gruyter. Bd. 1, S. 1418-1436.

Roelcke, Th. (1999): Fachsprachen. Berlin: Erich Schmidt.

Simonnæs, I. (2009): Verstehen und Interpretation in der intralingualen Rechtskommunikation. Voraussetzung und Anwendung in Theorie und Empirie. In: trans-kom 2(2), S. 160-172.

Tarantino, M. (2004), Epistemic and Dialectic Pathway to Knowledge, Meaning and Language Advancement. In: LSP \& Professional Communication 4 (1), S. 69-88. 